\title{
Does Higher Self-Reported Cardiorespiratory Fitness Reduce the Odds of Hospitalization From COVID-19?
}

\author{
Jason P. Brandenburg, Iris A. Lesser, Cynthia J. Thomson, and Luisa V. Giles
}

\begin{abstract}
Background: Regular physical activity and higher cardiorespiratory (CR) fitness enhance immune function, possibly reducing coronavirus disease 2019 (COVID-19) infection severity. The aim was to assess the association between physical activity and self-reported CR fitness on COVID-19 infection characteristics. Methods: Participants formerly testing positive for COVID19 completed an online questionnaire measuring COVID-19 infection characteristics and complications, self-reported CR fitness level, physical activity, and sociodemographic and health-related characteristics. Self-reported CR fitness was determined as the pace to cover $4.8 \mathrm{~km}$ without becoming overly fatigued (with slow walking, brisk walking, jogging, and running corresponding to low, moderate, good, and excellent levels of fitness, respectively). Results: A total of 263 individuals completed the survey. Compared with the lowest level of self-reported CR fitness, the odds of hospitalization significantly decreased by $64 \%$ (odds ratio $=0.36 ; 95 \%$ confidence interval, $0.13-0.98 ; P=.04$ ) in individuals reporting the ability to maintain a brisk walk. In individuals reporting the ability to maintain a jogging pace, the further reduction in hospitalization was not significant (odds ratio $=0.22 ; 95 \%$ confidence interval, $0.05-1.04 ; P=.05$ ). For COVID-19 symptom severity and number, there were no significant associations with self-reported CR fitness or physical activity levels. Conclusions: For individuals with low self-reported CR fitness, improving CR fitness represents a strategy to reduce the risk of hospitalization from COVID-19.
\end{abstract}

Keywords: coronavirus, exercise, physical activity, physical fitness

Coronavirus disease 2019 (COVID-19) is a highly infectious disease resulting from severe acute respiratory syndrome coronavirus 2 (SARS-CoV-2). COVID-19 was first observed in midDecember of 2019 in the city of Wuhan (China). ${ }^{1}$ As of fall 2020, COVID-19 has infected more than 100 million people in 220 different countries, resulting in more than 2 million deaths. ${ }^{2}$ Typically, infection with COVID-19 results in modest symptoms, the most common of which include coughing, fever/chills, sore throat, dyspnea, and myalgia. ${ }^{3,4}$ However, increased infection severity leading to poorer health outcomes is associated with older age and preexisting conditions, particularly diabetes, hypertension, chronic lung disease, and obesity. ${ }^{5,6}$

Currently, although vaccination plans in many countries have begun, control measures to protect against the spread of COVID-19 and manage the pandemic are still required and include proper handwashing, proper coughing etiquette, use of face coverings, physical distancing, and restrictions on nonessential travel. ${ }^{7}$ Less attention, however, has been given to the use of healthy lifestyle practices that improve immune functioning. ${ }^{7}$ Participation in regular exercise and physical activity, leading to improvements in physical fitness, may be one such behavior. It has previously been shown that regular physical activity and higher physical fitness levels enhance immune function ${ }^{8,9}$ and, therefore, might reduce the susceptibility to COVID-19 infection and infection severity. For example, a single bout of cardiorespiratory (CR) exercise results in immune cell mobilization, redistribution, and heightened cell activity, ${ }^{8,10}$ collectively increasing immunosurveillance and

Brandenburg, Lesser, and Giles are with the School of Kinesiology, University of the Fraser Valley, Chilliwack, BC, Canada. Thomson is with the School of Health Studies, University of the Fraser Valley, Chilliwack, BC, Canada. Brandenburg (jason.brandenburg@ufv.ca) is corresponding author. reducing susceptibility to, and severity of, viral infections. ${ }^{10,11}$ When repeated regularly, exercise and physical activity that improve physical fitness result in chronically higher levels of many immune cell markers, decreased incidence rates for influenza and pneumonia, improved immune response to vaccination, and reduced mortality rate from infection. ${ }^{12,13}$ In fact, during the 1997 Hong Kong flu epidemic, patients who reported higher levels of physical activity had a significantly lower risk of mortality than those who reported lower levels of physical activity.$^{14}$ In addition, engaging in regular physical activity and having higher levels of physical fitness reduce inflammatory biomarkers and slow the agerelated decline in immune function. ${ }^{15,16}$

This anti-inflammatory effect may be particularly relevant to individuals with comorbidities who, due to chronic low-grade inflammation, are at an elevated risk of hospitalization and mortality from COVID-19. ${ }^{16}$

Because of the recognized beneficial effects of physical activity on immunity, inflammation, and infection, ${ }^{9,11}$ it has been hypothesized that people who engage in regular physical activity and/or have higher levels of physical fitness will experience a less severe infection compared with those who are inactive or less fit. ${ }^{17}$ In an effort to curb the number of high-risk patients requiring hospitalization with COVID-19, the Center for Perioperative Care in the United Kingdom recommends that high-risk patients engage in brisk exercise in addition to other preventative health behaviors. ${ }^{18}$ Currently, however, the influence of physical activity and physical fitness levels on COVID-19 symptoms and outcomes has not been assessed. With the relatively high global prevalence of adults who do not meet the recommended amount of physical activity ( $23 \%$ of men and $32 \%$ of women) ${ }^{19}$ combined with the fact that the COVID-19 pandemic further reduces participation in physical activity, with or without lockdown measures in place, ${ }^{20,21}$ the conditions for more severe health complications and higher 
mortality rates from COVID-19 are apparent. Therefore, understanding the impact of physical activity and physical fitness on COVID-19 illness characteristics and complications could be beneficial in managing the global pandemic. Based on this, the purpose of this investigation is to understand whether physical activity and physical fitness levels are related to COVID-19 infection symptom characteristics and any adverse complications resulting from infection. We hypothesize that participants who report higher physical activity and physical fitness levels will have fewer symptoms, milder symptom severity, and fewer complications compared with those who engage in less physical activity and have lower levels of physical fitness.

\section{Methods}

\section{Population and Study Design}

Participants were recruited through social media platforms, including Facebook, Twitter, and Reddit. For Reddit, researchers searched for, and posted to, COVID-19 and coronavirus forums (or subreddits; eg, r/CoronavirusCanada, r/CoronavirusUK, etc). Recruitment of participants also occurred via an online directory that matched the researchers with potential volunteers residing in the province of British Columbia who satisfied the eligibility criteria and expressed an interest in participating in the current study (ReachBC). The 2 inclusion criteria were over the age of 18 years and a confirmed/positive COVID-19 test result. Participants provided online informed consent through Survey Monkey. The University of the Fraser Valley Human Research Ethics Board approved this study.

Over an approximate 3-month period (early July to midOctober), participants completed a questionnaire using an online survey platform (SurveyMonkey). The survey comprised 4 parts: confirmation of a positive COVID-19 test result, self-reported COVID-19 infection characteristics, and self-reported physical fitness and physical activity assessment as well as sociodemographic and health-related characteristics.

\section{COVID-19 Infection Characteristics}

To assess COVID-19 symptom number and severity, a 12-symptom influenza severity scale 22 was modified to include symptom characteristics of COVID-19 infection by adding 4 extra symptoms. Specifically, participants were asked about the presence of a dry cough, wheezing or difficulty breathing, nasal congestion, sore throat, ear pain, loss of smell or taste, fatigue, myalgia, feverishness, chills, headache, loss of appetite, vomiting, nausea, abdominal pain, and diarrhea. Each symptom was assessed using a 4-point scale $(0=$ absent, $1=$ mild, $2=$ moderate, and $3=$ severe $)$. Symptom number was determined by summing the number of symptoms that received a mild or higher rating in the 16-item symptom questionnaire in addition to any described symptoms beyond those listed. For COVID-19 severity, the ratings from the 16 symptoms were summed to form a symptom severity score that ranged from 1 (a single mild symptom) to 48 (all symptoms were severe). The median value for symptom number and symptom severity was used to dichotomize the variables for subsequent analyses. Participants were also asked to indicate whether their COVID-19 treatment required hospitalization (not including emergency room visits), admission to intensive care, and supportive care measures (eg, mechanical ventilation). Due to the high degree of redundancy between these items, only hospitalization was used in the analyses.

\section{Self-Reported CR Fitness and Physical Activity Assessment}

Self-reported CR fitness, prior to infection with COVID-19, was determined using a modified version of the perceived functional ability questionnaire. Instead of asking participants to rate their pace as slow, normal, or fast, the modified perceived functional ability questionnaire used a 13-point scale to ask participants to select how quickly they could walk, jog, or run a known distance (equal to $4.8 \mathrm{~km}$ or 3 miles) without becoming breathless or overly fatigued. With the assumption that individuals with higher selfreported CR fitness levels would cover $4.8 \mathrm{~km}$ using a faster pace, self-reported CR fitness was then grouped into 4 classifications: $>10 \mathrm{~min} / \mathrm{km}$ (ie, slow walking pace, representing low CR fitness), 10 to $>8.7 \mathrm{~min} / \mathrm{km}$ (ie, brisk walking pace, representing moderate CR fitness), 8.7 to $>6.2 \mathrm{~min} / \mathrm{km}$ (ie, jogging pace, representing good CR fitness), and 6.2 to $4.4 \mathrm{~min} / \mathrm{km}$ (ie, running pace, representing excellent $\mathrm{CR}$ fitness). The perceived functional ability questionnaire has been shown to accurately predict maximal oxygen uptake; however, the validity and reliability of the modifications used in the present study were not determined. ${ }^{23}$ Physical activity participation was assessed using the modified physical activity rating (PA-R) questionnaire, which asked participants to rate their physical activity level, using a 0 to 7 scale, over the 6 months prior to the COVID-19 diagnosis. ${ }^{23}$ Physical activity level was then categorized into 4 groupings: no regular physical activity (0-1), participation in moderate-intensity activity of any duration (2-3), regular participation in vigorousintensity activity for $<1$ hour per week (4-5), and regular participation in vigorous-intensity activity for a weekly duration greater than 1 hour $(6-7) .^{24}$

\section{Covariates}

Demographic and health-related covariates included age, sex, relationship status, ethnic origin, education, household income, smoking (current or nonsmoker; including cigarettes and vaping), alcohol consumption (never; weekly; daily; more than daily), body mass index (BMI; from self-reported height and body mass), and presence of physician-diagnosed COVID-19 comorbidities (including diabetes, asthma, chronic obstructive pulmonary disease, renal disease, hypertension, cardiovascular disease, and pregnancy). The time between the recovery from COVID-19 and completion of the online survey was also used as a covariate. Results from the analyses using this covariate are presented in the supplementary results file (see Supplementary Tables 1 and 2 [available online]).

\section{Statistical Analysis}

For each dependent variable, cross-tabulation with $\chi^{2}$ analysis was performed on each covariate and independent variable. Following this, a logistic regression (univariate analyses) was performed separately between each covariate variable and each dependent variable (COVID-19 symptom number, symptom severity, and hospitalization) to determine suitability for inclusion in the multivariate models. Based on these results, a multivariate logistic regression was performed with the significant covariates $(P<$ .05 ), each independent variable (self-reported CR fitness or physical activity), and each dependent variable. This analysis plan was similar to that used by Reinholdsson et al. ${ }^{25}$ Multiple linear regression results for symptom severity (as a continuous variable) are presented in the supplementary results file (see Supplementary 
Table 3 [available online]). IBM SPSS Statistics (version 25, Armonk, NY) was used for all statistical analyses.

\section{Results}

Descriptive data are presented as number (n) and percentage (\%) for all variables. About 424 participants initiated the online survey; however, only 316 participants reported a positive test result for COVID-19. After removal of participants with missing or incomplete data, 263 individuals were included in the analyses (Figure 1). Of the 263 participants, there were $150(57 \%)$ women and 113 $(43 \%)$ men. Eight-six percentage $(n=227)$ were under the age of 65 years, and $53(20 \%)$ were categorized as having obesity (BMI $\geq 30 \mathrm{~kg} / \mathrm{m}^{2}$ ). With respect to race/ethnic origin, $80 \%$ identified as being White/European, 9\% were Asian, 4\% were Latin American, $2 \%$ were African American, and $2 \%$ were Indigenous. Ninety-one percentage $(n=239)$ of participants resided in North America, 6\% $(\mathrm{n}=16)$ in Europe, $1.5 \%(\mathrm{n}=4)$ in South America, and $1.5 \%(\mathrm{n}=$ 4) in Asia or Africa. COVID-19 infection characteristics, CR fitness and physical activity characteristics, and other health-related variables can be found in Table 1 .

Multivariate logistic regression, controlling for age, BMI, and preexisting condition revealed a significant association between self-reported CR fitness and hospitalization due to COVID-19 (Table 2). Specifically, when the pace to cover a distance of $4.8 \mathrm{~km}$ increased from a slow pace (reference $=$ low CR fitness) to a brisk pace (moderate CR fitness), the odds of hospitalization decreased by $64 \%$ (odds ratio $=0.36 ; 95 \%$ confidence interval, $0.13-0.98, P=.04)$. A trend was observed for a greater reduction in hospitalization due to COVID-19 when the pace to cover $4.8 \mathrm{~km}$ increased to a jog (good CR fitness; odds ratio $=0.22 ; 95 \%$ confidence interval, $0.05-1.04 ; P=.05$ ). For COVID-19 severity and symptom number, there were no significant associations with self-reported CR fitness or physical activity levels (Tables 3 and 4).

\section{Discussion}

To our knowledge, this is the first study to report on the association between self-reported CR fitness, physical activity, and COVID-19 disease severity. We hypothesized that individuals who reported higher physical activity and physical fitness levels would experience fewer symptoms, milder symptom severity, and fewer complications compared with individuals who engaged in less physical activity and had lower self-reported levels of physical fitness. The primary finding was that a small increase in self-reported CR fitness level (as indicated by the ability to cover $4.8 \mathrm{~km}$ using a brisk walking pace vs a slow pace) significantly reduced the odds of hospitalization due to COVID-19 by 64\%. A larger increase in self-reported CR fitness (as indicated by the ability to cover $4.8 \mathrm{~km}$ using a jogging pace) further decreased the chance of COVID-19-related hospitalization (by 78\%); however, this was not significant $(P=.05)$.

The findings of the present study are in line with those of Brawner et al, ${ }^{26}$ who observed an inverse association between peak metabolic equivalents (METs) attained during a symptom-limited treadmill stress test and hospitalization from COVID-19 in 246 patients who tested positive with COVID-19.

Specifically, for every unit increase in peak METs, a $13 \%$ reduction in hospitalization was observed. Given that a significant dose-response relationship between CR fitness and a reduction in the rate of hospitalization was observed by Brawner et al, ${ }^{26}$ it was unexpected that the odds reduction yielded by a further increase in self-reported CR fitness (the ability to cover $4.8 \mathrm{~km}$ using a jogging pace) only approached significance $(P=.05)$. This inconsistency is likely explained by the difference in the participant characteristics between the 2 studies. In the study by Brawner et al, ${ }^{26}$ participants were less healthy than those of the current study as indicated by lower CR fitness (mean =7.5 [2.8] METs), older age $($ mean $=59$ [12] y), a higher BMI (mean = 32 [7]), and increased likelihood of having single and multiple high-risk COVID-19 comorbidities. Considering this, the relatively healthy status of the participants of the current study likely accounted for the lack of a significant odds reduction of hospitalization at the higher self-reported fitness level (the ability to cover $4.8 \mathrm{~km}$ using a jogging pace).

Practically speaking, it is important to highlight that the protective association between self-reported CR fitness and reduced COVID-19-related hospitalization was observed at the low end of the self-reported CR fitness scale. This means that individuals with low levels of CR fitness only need to experience small improvements in CR fitness to significantly reduce the odds of hospitalization if they become infected with COVID-19. With up to $5 \%$ of individuals infected with COVID-19 becoming critically ill, ${ }^{27}$ the potential to exceed the critical care capacity of hospitals is possible as COVID-19 cases climb. Furthermore, approximately $54 \%$ of patients hospitalized due to COVID-19 have been reported to experience respiratory failure and more than $30 \%$ require mechanical ventilation. ${ }^{28}$ Therefore, any protective mechanism reducing COVID-19 hospitalization is of utmost importance. The findings from the current study suggest that improving CR fitness, even by a small amount in those with low levels of

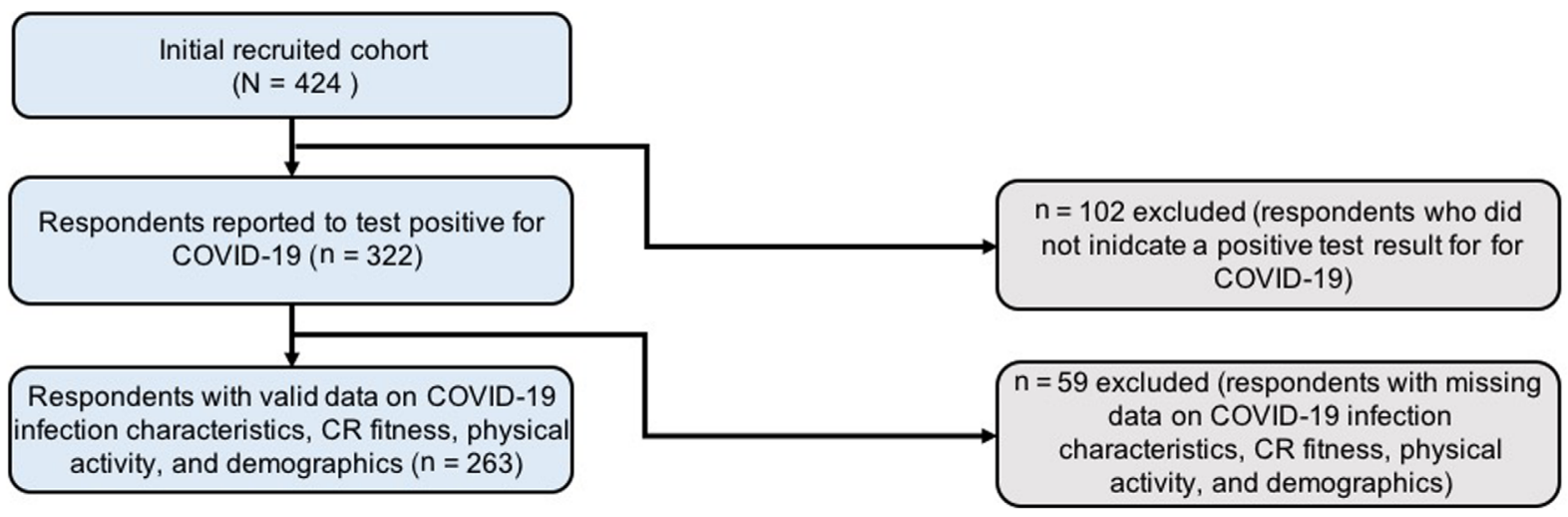

Figure 1 - Flowchart description of the participants included in the study population. CR indicates cardiorespiratory; COVID-19, coronavirus disease 2019. 
Table 1 COVID-19 Infection, CR Fitness and Physical Activity Characteristics, and Other Health-Related Characteristics of the Participants

\begin{tabular}{|c|c|c|c|}
\hline & $\begin{array}{c}\text { Total } \\
(\mathrm{n}=263)\end{array}$ & $\begin{array}{l}\text { Females } \\
(n=150)\end{array}$ & $\begin{array}{c}\text { Males } \\
(n=113)\end{array}$ \\
\hline \multicolumn{4}{|l|}{ Number of COVID-19 symptoms } \\
\hline Less ( $\leq 9$ symptoms) & $157(60 \%)$ & $80(30 \%)$ & $77(29 \%)$ \\
\hline More ( $\geq 10$ symptoms) & $106(40 \%)$ & $70(27 \%)$ & $36(13 \%)$ \\
\hline \multicolumn{4}{|l|}{ Modified influenza severity score } \\
\hline Less severe ( $\leq 33$ symptoms) & $140(53 \%)$ & $71(27 \%)$ & $69(26 \%)$ \\
\hline More severe ( $\geq 34$ symptoms) & $123(47 \%)$ & $79(30 \%)$ & $44(17 \%)$ \\
\hline \multicolumn{4}{|l|}{ Hospitalization due to COVID-19 } \\
\hline Yes & $28(11 \%)$ & $11(4 \%)$ & $17(7 \%)$ \\
\hline No & $235(89 \%)$ & $139(52 \%)$ & $96(37 \%)$ \\
\hline \multicolumn{4}{|l|}{ CR fitness, $\mathrm{min} / \mathrm{km}$} \\
\hline$>10$ & $34(13 \%)$ & $21(8 \%)$ & $13(5 \%)$ \\
\hline $8.7-10$ & $97(37 \%)$ & $59(22 \%)$ & $38(15 \%)$ \\
\hline $8.7-6.2$ & $80(30 \%)$ & $49(18 \%)$ & $31(12 \%)$ \\
\hline $6.2-4.4$ & $52(20 \%)$ & $21(8 \%)$ & $31(12 \%)$ \\
\hline \multicolumn{4}{|l|}{ Physical activity } \\
\hline No/low & $39(15 \%)$ & $21(8 \%)$ & $18(7 \%)$ \\
\hline Moderate & $73(28 \%)$ & $45(17 \%)$ & $28(11 \%)$ \\
\hline$<1 \mathrm{~h}$ of vigorous & $49(18 \%)$ & $31(11 \%)$ & $18(7 \%)$ \\
\hline$>1 \mathrm{~h}$ of vigorous & $102(39 \%)$ & $53(20 \%)$ & $49(19 \%)$ \\
\hline \multicolumn{4}{|l|}{ Age } \\
\hline$<65$ & $227(86 \%)$ & $133(50 \%)$ & $94(36 \%)$ \\
\hline$\geq 65$ & $36(14 \%)$ & $17(7 \%)$ & $19(7 \%)$ \\
\hline \multicolumn{4}{|l|}{ BMI, $\mathrm{kg} / \mathrm{m}^{2}$} \\
\hline$<30$ & $210(80 \%)$ & $118(45 \%)$ & $92(35 \%)$ \\
\hline$\geq 30$ & $53(20 \%)$ & $32(12 \%)$ & $21(8 \%)$ \\
\hline \multicolumn{4}{|c|}{ Presence of high-risk comorbidities ${ }^{\mathrm{a}}$} \\
\hline Yes & $109(41 \%)$ & $62(24 \%)$ & $47(18 \%)$ \\
\hline No & $154(59 \%)$ & $88(33 \%)$ & $66(25 \%)$ \\
\hline \multicolumn{4}{|l|}{ Tobacco use (eg, smoking, vaping) } \\
\hline Yes & $15(6 \%)$ & $6(2 \%)$ & $9(3 \%)$ \\
\hline No & $248(94 \%)$ & $144(55 \%)$ & $104(39 \%)$ \\
\hline \multicolumn{4}{|l|}{ Alcohol consumption } \\
\hline Never & $108(41 \%)$ & $64(24 \%)$ & $44(17 \%)$ \\
\hline Rarely & $75(28 \%)$ & $49(18 \%)$ & $26(10 \%)$ \\
\hline Moderately & $60(23 \%)$ & $32(12 \%)$ & $28(11 \%)$ \\
\hline More than daily & $20(7 \%)$ & $5(2 \%)$ & $15(5 \%)$ \\
\hline
\end{tabular}

Abbreviations: BMI, body mass index; CR, cardiorespiratory; COVID-19, coronavirus disease 2019; COPD, chronic obstructive pulmonary disease.

${ }^{a}$ High-risk comorbidities including diabetes mellitus, asthma, COPD, renal disease, hypertension, cardiovascular disease, and pregnancy.

self-reported fitness, appears to be an effective strategy to reduce COVID-19 hospitalizations.

Although not the purpose of the current study, there are plausible physiological mechanisms accounting for the protective effect of higher CR fitness levels. Such mechanisms include lower levels of inflammation, improved inflammatory and immune signaling, augmented immune function, reduction in comorbidities, and reduced stress. ${ }^{11,15}$ Despite all the possible mechanisms underlying increased CR fitness and better immune function, the association between self-reported CR fitness and symptom severity and symptom number was not significant. One reason for this could be due to the limitations in objectively assessing COVID-19 severity. Although we modified the influenza severity scale ${ }^{22}$ to include symptoms consistent with COVID-19, severity score was influenced by the ability of the participant to accurately recall symptoms, the number of symptoms, and symptom experience, with the latter being subjective. On average, the time between recovery from COVID-19 and completion of the online survey was 91 days. It is possible this time frame influenced the accuracy of symptom recall. Hospitalization, on the other hand, was not subjective, and this may have contributed to the significant association between selfreported CR fitness and reduced hospitalization. 
Table 2 Logistic Regression Results for the Associations Between CR Fitness and Physical Activity and the Number of COVID-19 Symptoms (Adjusted for Age and $\operatorname{Sex}^{\mathrm{a}, \mathrm{b}}$ )

\begin{tabular}{lccccc}
\hline Variable & B (SE) & Wald & OR & 95\% CI & $\boldsymbol{P}$ value \\
\hline CR fitness ${ }^{\mathrm{a}}, \mathrm{min} / \mathrm{km}$ & & & & & \\
$>10$ & $\mathrm{REF}$ & $\mathrm{REF}$ & $\mathrm{REF}$ & & \\
$8.7-10$ & $-0.3(.4)$ & 0.5 & 0.73 & $0.32-1.68$ & .46 \\
$8.7-6.2$ & $-0.6(.4)$ & 1.7 & 0.56 & $0.23-1.33$ & .19 \\
$6.2-4.4$ & $-1.0(.5)$ & 3.9 & 0.38 & $0.15-0.99$ & .05 \\
Physical activity & & & & & \\
No/low & $\mathrm{REF}$ & $\mathrm{REF}$ & $\mathrm{REF}$ & & \\
Moderate & $0.5(.4)$ & 1.5 & 1.67 & $0.74-3.80$ & .8 \\
$<1$ h of vigorous & $0.1(.4)$ & 0 & 1.08 & $0.45-2.60$ & .6 \\
$>1$ h of vigorous & $-0.6(.4)$ & 2.1 & 0.56 & $0.26-1.23$ & .14 \\
\hline
\end{tabular}

Abbreviations: CI, confidence interval; CR, cardiorespiratory; COVID-19, coronavirus disease 2019; OR, odds ratio; $\mathrm{REF}$, reference value. ${ }^{\mathrm{a}} \mathrm{Age}: \mathrm{OR}=1.65$, $P=.06$; sex: $\mathrm{OR}=0.294, P=.002 .{ }^{\mathrm{b}}$ Age: $\mathrm{OR}=0.28, P=.004$; sex: $\mathrm{OR}=1.69$, $P=.051$.

Table 3 Logistic Regression Results for the Associations Between CR Fitness and Physical Activity and the Severity of COVID-19 Symptoms (Adjusted for BMI, Preexisting Condition, and Sex ${ }^{a, b}$ )

\begin{tabular}{lccccc}
\hline Variable & B (SE) & Wald & OR & $\mathbf{9 5 \% ~ C l}$ & $\boldsymbol{P}$ value \\
\hline CR fitness $^{\mathrm{a}}$, min/km & & & & & \\
$>10$ & REF & REF & REF & & \\
$8.7-10$ & $-0.5(0.4)$ & 0 & 0.96 & $0.42-$ & .9 \\
& & & & 2.17 & \\
$8.7-6.2$ & $0.4(0.5)$ & 0 & 1.04 & $0.42-$ & .9 \\
& & & & 2.58 & \\
$6.2-4.4^{\mathrm{c}}$ & $-0.3(0.5)$ & 0.3 & 0.76 & $0.28-$ & .6 \\
& & & & 2.04 & \\
Physical activity & & & & & \\
No/low & REF & REF & REF & & \\
Moderate & $0.3(0.4)$ & 0.4 & 1.30 & $0.58-$ & .5 \\
& & & & 2.90 & \\
$<1$ h of vigorous & $0.4(0.5)$ & 0.6 & 1.43 & $0.57-$ & .4 \\
& & & & 3.56 & \\
$>1$ h of vigorous & $-0.4(0.4)$ & 1.0 & 0.65 & $0.28-$ & .3 \\
& & & & 1.48 & \\
\hline
\end{tabular}

Abbreviations: BMI, body mass index; CI, confidence interval; CR, cardiorespiratory; COVID-19, coronavirus disease 2019; OR, odds ratio; REF, reference value; $\mathrm{SE}$, standard error.

${ }^{a} \mathrm{BMI}: \mathrm{OR}=1.58, P=.17$; sex: $\mathrm{OR}=1.68, P=.048$; preexisting condition: $\mathrm{OR}=1.61, P=.09$. ${ }^{\mathrm{b}} \mathrm{BMI}$ : $\mathrm{OR}=1.48, P=.25$; sex $\mathrm{OR}=1.65, P=.057$; preexisting condition: $\mathrm{OR}=0.44, P=.19$.

Despite the numerous acute immune and anti-inflammatory benefits from performing a bout of physical activity, ${ }^{7,15}$ the current study did not observe a significant association between physical activity and any of the COVID-19 infection characteristics. On the surface, these findings are inconsistent with the recent observations indicating that physical inactivity was a lifestyle factor associated with hospitalization due to COVID-19. ${ }^{29}$ However, this study only examined the lifestyle factors in patients hospitalized due to
Table 4 Logistic Regression Results for the Associations Between CR Fitness, Physical Activity and Physical Fitness, and Hospitalization due to COVID-19 (Adjusted for Age, BMI, and Preexisting Condition ${ }^{\mathrm{a}, \mathrm{b}}$ )

\begin{tabular}{lccccc}
\hline Variable & B (SE) & Wald & OR & $95 \%$ Cl & $\begin{array}{c}\boldsymbol{P} \\
\text { value }\end{array}$ \\
\hline $\begin{array}{l}\text { CR fitness } \\
\text { km }\end{array}$ & & & & & \\
$\quad>10$ & REF & REF & REF & & \\
$8.7-10$ & $-1.02(0.5)$ & 4.0 & 0.36 & $0.13-0.98$ & .04 \\
$8.7-6.2$ & $-1.5(0.7)$ & 3.8 & 0.22 & $0.05-1.02$ & .05 \\
$6.2-4.4^{\mathrm{c}}$ & -19.4 & - & - & - & - \\
& $(5580)$ & & & & \\
Physical activity & & & & & \\
$\quad$ No/low & $\mathrm{REF}$ & $\mathrm{REF}$ & $\mathrm{REF}$ & & \\
$\quad$ Moderate & $0.4(0.6)$ & 0.3 & 1.43 & $0.42-4.84$ & .6 \\
$<1$ h of vigorous & $0.5(0.8)$ & 0 & 1.05 & $0.22-5.00$ & .9 \\
$>1$ h of vigorous & $0.7(0.8)$ & 0 & 1.06 & $0.23-4.79$ & .6 \\
\hline
\end{tabular}

Abbreviations: BMI, body mass index; CI, confidence interval; $\mathrm{CR}$, cardiorespiratory; COVID-19, coronavirus disease 2019; OR, odds ratio; REF, reference value.

${ }^{\mathrm{a}} \mathrm{BMI}: \mathrm{OR}=1.58, P=.35$; age: $\mathrm{OR}=5.22, P=.001$; preexisting condition: $\mathrm{OR}=2.26, P=.121 .{ }^{\mathrm{b}} \mathrm{BMI} \mathrm{OR}=2.08 P=.15$; age: $\mathrm{OR}=7.57, P<.001$; preexisting condition: $\mathrm{OR}=3.5, P=.014$. ${ }^{\mathrm{C}}$ Insufficient sample size to generate results.

COVID-19 and made no comparisons with nonhospitalized cases of COVID-19, thus making it difficult to ascertain the role of physical activity in protecting against hospitalization. Furthermore, the study did not include any indicator of CR fitness in the hospitalized COVID-19 cases. $^{29}$ It is possible that the lack of a significant association between physical activity and COVID-19 infection characteristics in the current study could be attributed to the healthy nature of the study participants relative to the general population. For example, in the current study, there was a relatively low prevalence of smoking, alcohol consumption, obesity, and number of participants above 65 years of age. The mean selfreported CR fitness level (equal to a pace of $8 \mathrm{~min} / \mathrm{km}$ ) combined with the fact that almost $60 \%$ of participants reported regularly participating in vigorous activity also indicated that the study population was more fit and active than the general population. Perhaps with a population more representative of the general population, the associations between physical activity and COVID-19 infection characteristics may have been stronger.

As previously indicated, a limitation of the current study was that the participants appeared to be healthier, more fit, and more active than the general population. This may have been due to participant recruitment strategies, such as social media platforms, which tend to target younger participants who may have higher fitness and physical activity levels ${ }^{30}$ and, as a result, raise the potential for volunteer bias. In addition, the accuracy of the selfreported CR fitness measure would have been influenced by the experience with continuous aerobic exercise as well as the experience with walking/jogging as both of these influence the ability to accurately estimate the pace to cover $4.8 \mathrm{~km}$. Despite these limitations, the findings from the present study, indicating that increases in self-reported CR fitness are associated with decreased odds of COVID-19 hospitalization, are important as many countries navigate the COVID-19 pandemic in the face of rising infection rates, the reimplementation of COVID-19 restrictions 
(eg, sheltering in place), and the potential for reductions in physical activity leading to decreasing CR fitness levels.

\section{Conclusions}

The odds of hospitalization due to COVID-19 were significantly reduced with an increase in self-reported CR fitness equal to the ability to cover $4.8 \mathrm{~km}$ using a brisk walking pace in comparison with a slow pace. Reductions in hospitalization from larger increases in self-reported CR fitness were not significant, and a relatively healthy participant pool likely accounted for this. There were no other significant associations between self-reported CR fitness or physical activity with self-reported severity and symptom number. Practically speaking, for individuals with low CR fitness, improving CR fitness represents a potential strategy that can reduce the risk of hospitalization from COVID-19.

\section{References}

1. Lake MA. What we know so far: COVID-19 current clinical knowledge and research. Clin Med J R Coll Physicians Lond. 2020; 20(2):124-127. doi:10.7861/clinmed.2019-coron

2. World Health Organization. Coronavirus disease (COVID-19) pandemic. https://www.who.int/emergencies/diseases/novel-coronavirus2019. Accessed November 15, 2020.

3. Zheng J. SARS-coV-2: an emerging coronavirus that causes a global threat. Int J Biol Sci. 2020;16(10):1678-1685. PubMed ID: 32226285 doi:10.7150/ijbs. 45053

4. Krajewska J, Krajewski W, Zub K, Zatoński T. COVID-19 in otolaryngologist practice: a review of current knowledge. Eur Arch Oto-Rhino-Laryngology. 2020;277(7):1885-1897. doi:10. 1007/s00405-020-05968-y

5. Wang B, Li R, Lu Z, Huang Y. Does comorbidity increase the risk of patients with covid-19: evidence from meta-analysis. Aging. 2020; 12(7):6049-6057. doi:10.18632/aging.103000

6. Long JD, Ward CA, Khorasani-Zadeh A. The impact of obesity on COVID-19 disease severity. PRiMER. 2020;4:5. doi:10.22454/ PRiMER.2020.104798

7. Nieman DC. Coronavirus disease-2019: a tocsin to our aging, unfit, corpulent, and immunodeficient society. J Sport Heal Sci. 2020;9(4): 293-301. doi:10.1016/j.jshs.2020.05.001

8. Duggal NA, Niemiro G, Harridge SDR, Simpson RJ, Lord JM. Can physical activity ameliorate immunosenescence and thereby reduce age-related multi-morbidity? Nat Rev Immunol. 2019; 19(9):563-572. PubMed ID: 31175337 doi:10.1038/s41577019-0177-9

9. Simpson RJ, Kunz H, Agha N, Graff R. Exercise and the regulation of immune functions. Prog Mol Biol Transl Sci. 2015;135:355-80. PubMed ID: 26477922 doi:10.1016/bs.pmbts.2015.08.001

10. Simpson RJ, Katsanis E. The immunological case for staying active during the COVID-19 pandemic. Brain Behav Immun. 2020;87:6-7. PubMed ID: 32311497 doi:10.1016/j.bbi.2020.04.041

11. Nieman DC, Wentz LM. The compelling link between physical activity and the body's defense system. J Sport Heal Sci. 2019;8(3): 201-217. doi:10.1016/j.jshs.2018.09.009

12. Wong GCL, Narang V, Lu Y, et al. Hallmarks of improved immunological responses in the vaccination of more physically active elderly females. Exerc Immunol Rev. 2019;25:20-33. PubMed ID: 30753128

13. Schuler PB, Leblanc PA, Marzilli TS. Effect of physical activity on the production of specific antibody in response to the 1998-99 influenza virus vaccine in older adults. J Sports Med Phys Fitness. 2003;43(3):404. PubMed ID: 14625524

14. Wong CM, Lai HK, Ou CQ, et al. Is exercise protective against influenza-associated mortality? PLoS One. 2008;3(5):e2108. PubMed ID: 18461130 doi:10.1371/journal.pone.0002108

15. Hjelstuen A, Anderssen SA, Holme I, Seljeflot I, Klemsdal TO. Markers of inflammation are inversely related to physical activity and fitness in sedentary men with treated hypertension. Am J Hypertens. 2006;19(7):669-675. PubMed ID: 16814119 doi:10.1016/j. amjhyper.2005.11.012

16. Zbinden-Foncea H, Francaux M, Deldicque L, Hawley JA. Does high cardiorespiratory fitness confer some protection against proinflammatory responses after infection by SARS-CoV-2? Obesity. 2020;28(8):1378-1381. PubMed ID: 32324968 doi:10.1002/oby. 22849

17. Sallis JF, Adlakha D, Oyeyemi A, Salvo D. An international physical activity and public health research agenda to inform coronavirus disease-2019 policies and practices. J Sport Heal Sci. 2020;9(4):328334. doi:10.1016/j.jshs.2020.05.005

18. Ahmed I. COVID-19-does exercise prescription and maximal oxygen uptake $\left(\mathrm{VO}_{2} \mathrm{max}\right)$ have a role in risk-stratifying patients? Clin Med J R Coll Physicians Lond. 2020;20(3):282-284. doi:10. 7861/clinmed.2020-0111

19. World Health Organization. Health topics: physical activity. https:// www.who.int/gho/ncd/risk_factors/physical_activity_text/en/ Accessed November 1, 2020.

20. Di Sebastiano KM, Chulak-Bozzer T, Vanderloo LM, Faulkner G. Don't walk so close to me: physical distancing and adult physical activity in Canada. Front Psychol. 2020;11:1895. PubMed ID: 32849110 doi:10.3389/fpsyg.2020.01895

21. Lesser IA, Nienhuis CP. The impact of COVID-19 on physical activity behavior and well-being of Canadians. Int J Environ Res Public Health. 2020;17(11):3899. doi:10.3390/ijerph17113899

22. Sokolow LZ, Naleway AL, Li DK, et al. Severity of influenza and noninfluenza acute respiratory illness among pregnant women, 20102012. Am J Obstet Gynecol. 2015;212(2):202.e1-202.e11. doi:10. 1016/j.ajog.2014.08.004

23. Bradshaw DI, George JD, Hyde A, et al. An accurate vo2max nonexercise regression model for 18-65-year-old adults. Res $Q$ Exerc Sport. 2005;76(4):426-432. PubMed ID: 16739680 doi:10.1080/ 02701367.2005.10599315

24. Matthews CE, Heil DP, Freedson PS, Pastides H. Classification of cardiorespiratory fitness without exercise testing. Med Sci Sports Exerc. 1999;31(3):486-493. PubMed ID: 10188755 doi:10.1097/ 00005768-199903000-00019

25. Reinholdsson M, Palstam A, Sunnerhagen KS. Prestroke physical activity could influence acute stroke severity (part of PAPSIGOT). Neurology. 2018;91(16):e1461-e1467. PubMed ID: 30232251 doi:10.1212/WNL.0000000000006354

26. Brawner CA, Ehrman JK, Bole S, et al. Maximal exercise capacity is inversely related to hospitalization secondary to coronavirus disease 2019. Mayo Clin Proc. 2020;96(1):P32-P39. doi:10.1016/j.mayocp. 2020.10.003

27. Razai MS, Doerholt K, Ladhani S, Oakeshott P. Coronavirus disease 2019 (COVID-19): a guide for UK GPS. BMJ. 2020;368:m800. doi:10.1136/bmj.m800 
28. Li X, Xu S, Yu M, et al. Risk factors for severity and mortality in adult COVID-19 inpatients in Wuhan. J Allergy Clin Immunol. 2020; 146(1):110-118. PubMed ID: 32294485 doi:10.1016/j.jaci.2020. 04.006

29. Hamer M, Kivimäki M, Gale CR, Batty GD. Lifestyle risk factors, inflammatory mechanisms, and COVID-19 hospitalization: a community-based cohort study of 387,109 adults in UK. Brain Behav Immun. 2020;87:184-187. PubMed ID: 32454138 doi:10. 1016/j.bbi.2020.05.059

30. Shatz I. Fast, free, and targeted: reddit as a source for recruiting participants online. Soc Sci Comput Rev. 2017;35(4):537-549. doi: $10.1177 / 0894439316650163$ 